\title{
A RECOMBINATION ALGORITHM FOR THE DECOMPOSITION OF MULTIVARIATE RATIONAL FUNCTIONS
}

\author{
GUILLAUME CHÈZE
}

\begin{abstract}
In this paper we show how we can compute in a deterministic way the decomposition of a multivariate rational function with a recombination strategy. The key point of our recombination strategy is the use of Darboux polynomials. We study the complexity of this strategy and we show that this method improves the previous ones. In the appendix, we explain how the strategy proposed recently by J. Berthomieu and G. Lecerf for the sparse factorization can be used in the decomposition setting. Then we deduce a decomposition algorithm in the sparse bivariate case and we give its complexity.
\end{abstract}

\section{INTRODUCTION}

The decomposition of univariate polynomials has been widely studied since 1922 (see [Rit22]), and efficient algorithms are known (see [AT85, BZ85, KL89, Gat90a, Gat90b, Gie88, Klü99]). There also exist results and algorithms in the multivariate case Dic87, Gat90a, Gie88, GGR03.

The decomposition of rational functions has also been studied; see Gie88, Zip91, AGR95, GW95. In the multivariate case the situation is the following:

Let $f\left(X_{1}, \ldots, X_{n}\right)=f_{1}\left(X_{1}, \ldots, X_{n}\right) / f_{2}\left(X_{1}, \ldots, X_{n}\right) \in \mathbb{K}\left(X_{1}, \ldots, X_{n}\right)$ be a rational function, where $\mathbb{K}$ is a field and $n \geq 2$. It is commonly said to be composite if it can be written $f=u(h)$ where $h\left(X_{1}, \ldots, X_{n}\right) \in \mathbb{K}\left(X_{1}, \ldots, X_{n}\right)$ and $u \in \mathbb{K}(T)$ such that $\operatorname{deg}(u) \geq 2$ (recall that the degree of a rational function is the maximum of the degrees of its numerator and denominator after reduction); otherwise $f$ is said to be non-composite. We remark that $\operatorname{deg} u \geq 2$ because univariate rational functions with degree equal to 1 are invertible for the composition. In this paper, we give an algorithm which computes a non-composite rational function $h \in \mathbb{K}\left(X_{1}, \ldots, X_{n}\right)$ and a rational function $u \in \mathbb{K}(T)$ such that $f=u(h)$.

In Chè10, the author shows that we can reduce the decomposition problem to a factorization problem and gives a probabilistic and a deterministic algorithm. The probabilistic algorithm is nearly optimal: it performs $\tilde{\mathcal{O}}\left(d^{n}\right)$ arithmetic operations. The deterministic one computes $\mathcal{O}\left(d^{2}\right)$ absolute factorizations and then performs $\tilde{\mathcal{O}}\left(d^{n+\omega+2}\right)$ arithmetic operations, where $d$ is the degree of $f$ and $\omega$ is the feasible matrix multiplication exponent as defined in [GG03, Chapter 12]. We recall that $2 \leq \omega \leq 2.376$. As in Chè10, we suppose in this work that $d$ tends to infinity and $n$ is fixed. We use the classical $\mathcal{O}$ and $\tilde{\mathcal{O}}$ ("soft $\mathcal{O}$ ") notation in the neighborhood of infinity as defined in [GG03, Chapter 25.7]. Informally speaking, "soft $\mathcal{O}$ "s are used for readability in order to hide logarithmic factors in complexity estimates.

Received by the editor November 3, 2010 and, in revised form, November 22, 2011.

2010 Mathematics Subject Classification. Primary 11Y16, 68W30; Secondary 12Y05, 12D05, $13 \mathrm{P} 05$.

(C) 2012 American Mathematical Society 1793 
In this paper we improve the complexity of the deterministic algorithm. With this algorithm we just compute two factorizations in $\mathbb{K}\left[X_{1}, \ldots, X_{n}\right]$ and then we use a recombination strategy. Under some hypotheses this new method performs $\tilde{\mathcal{O}}\left(d^{n+\omega-1}\right)$ arithmetic operations.

The decomposition of multivariate rational functions appears when we study the kernel of a derivation; see MO04]. In [MO04 the author uses Darboux polynomials and gives an algorithm which works with $\tilde{\mathcal{O}}\left(d^{\omega n}\right)$ arithmetic operations.

In this paper, we are also going to use Darboux polynomials (see Section 1 for a definition) and we add a recombination strategy. Roughly speaking, we are going to factorize the numerator and the denominator and then thanks to a property of Darboux polynomials we are going to show that we can recombine the factors and deduce the decomposition. More precisely, irreducible factors of the numerator and the denominator do not give the rational function $h$, but if we use Darboux polynomials we can put them together in order to deduce $h$.

The decomposition of multivariate rational functions also appears when we study intermediate fields of an unirational field and the extended Lüroth's Theorem (see GRS01, Chè10]) and when we study the spectrum of a rational function (see Chè10 and the references therein).

The study of decomposition is an active area of research: for a study on multivariate polynomial systems see e.g. [FP09, [FGP10], for a study on symbolic polynomials see e.g. Wat09, for a study on Laurent polynomials see e.g. Wat08, for effective results on the reduction modulo a prime number of a non-composite polynomial or a rational function see e.g. [CN10, BDN09, BCN], for combinatorial results see e.g. Gat11.

In this paper, we improve the strategy proposed in MO04. As in MO04, we consider fields with characteristic zero. Furthermore, as we want to give a precise complexity estimate we are going to suppose that:

Hypothesis (C):

$\mathbb{K}$ is a number field: $\mathbb{K}=\mathbb{Q}[\alpha], \alpha$ is an algebraic number of degree $r$.

As in [Chè10, we are going to suppose that the following hypothesis is satisfied:

Hypothesis $(\mathrm{H})$ :

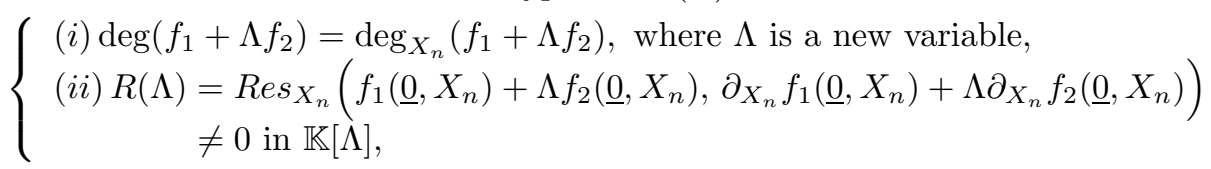

where $\operatorname{deg}_{X_{n}} f$ represents the partial degree of $f$ in the variable $X_{n}$, $\operatorname{deg} f$ is the total degree of $f$ and $\operatorname{Res}_{X_{n}}$ denotes the resultant relative to the variable $X_{n}$.

This hypothesis is not restrictive but is necessary, because we will use the factorization algorithms proposed in [Lec07, where this kind of hypothesis is needed. Actually, in [Lec07] the author studies the factorization of a polynomial $F$ and uses hypothesis $(\mathrm{L})$, where $(\mathrm{L})$ is the following:

\section{Hypothesis (L):}

$$
\left\{\begin{array}{l}
(i) \operatorname{deg}_{X_{n}} F=\operatorname{deg} F, \text { and } F \text { is monic in } X_{n}, \\
(i i) \operatorname{Res}_{X_{n}}\left(F\left(\underline{0}, X_{n}\right), \frac{\partial F}{\partial X_{n}}\left(\underline{0}, X_{n}\right)\right) \neq 0 .
\end{array}\right.
$$

If $F$ is squarefree, then hypothesis (L) is not restrictive since it can be assured by means of a generic linear change of variables, but we will not discuss this question here (for a complete treatment in the bivariate case, see [CL07, Proposition 1]). 
Roughly speaking, our hypothesis $(\mathrm{H})$ is the hypothesis $(\mathrm{L})$ applied to the polynomial $f_{1}+\Lambda f_{2}$. In $(\mathrm{H}, i)$ we do not assume that $f_{1}+\Lambda f_{2}$ is monic in $X_{n}$. Indeed, the leading coefficient relative to $X_{n}$ can be written: $a+\Lambda b$, with $a, b \in \mathbb{K}$. In our algorithm, we evaluate $\Lambda$ to $\lambda$ such that $a+\lambda b \neq 0$. Then we can consider the monic part of $f_{1}+\lambda f_{2}$ and we get a polynomial satisfying $(\mathrm{L}, i)$. Then $(\mathrm{H}, i)$ is sufficient in our situation. Furthermore, in this paper, we assume $f_{1} / f_{2}$ to be reduced, i.e., $f_{1}$ and $f_{2}$ are coprime. We recall in Lemma 9 that in this situation $f_{1}+\Lambda f_{2}$ is squarefree. Thus hypothesis $(\mathrm{H})$ is not restrictive.

Furthermore, hypothesis $(\mathrm{H})$ will also be useful in a preprocessing step; see Section 2. In this preprocessing step we reduce the decomposition to two factorizations of squarefree polynomials.

Complexity model. In this paper the complexity estimates charge a constant cost for each arithmetic operation $(+,-, \times, \div)$ and the equality test. All the constants in the base fields are thought to be freely at our disposal.

In this paper we suppose that the number of variables $n$ is fixed and that the degree $d$ tends to infinity.

Polynomials are represented by dense vectors of their coefficients in the usual monomial basis. For each integer $d$, we assume that we are given a computation tree that computes the product of two univariate polynomials of degree at most $d$ with at most $\tilde{\mathcal{O}}(d)$ operations, independently of the base ring; see [GG03, Theorem 8.23]. Then with a Kronecker substitution we can compute the product of two multivariate polynomials with degree $d$ with $n$ variables with $\tilde{\mathcal{O}}\left(d^{n}\right)$ arithmetic operations. We also recall (see GG03, Corollary 11.8]) that if $\mathbb{K}$ is an algebraic extension of $\mathbb{Q}$ of degree $r$, then each field operation in $\mathbb{K}$ takes $\tilde{\mathcal{O}}(r)$ arithmetic operations in $\mathbb{Q}$.

We use the constant $\omega$ to denote a feasible matrix multiplication exponent as defined in GG03, Chapter 12]: two $n \times n$ matrices over $\mathbb{K}$ can be multiplied with $\mathcal{O}\left(n^{\omega}\right)$ field operations. As in BP94 we recall that $2 \leq \omega \leq 2.376$ and that the computation of a solution basis of a linear system with $m$ equations and $d \leq m$ unknowns over $\mathbb{K}$ takes $\mathcal{O}\left(m d^{\omega-1}\right)$ operations in $\mathbb{K}$ [BP94, Chapter 2] (see also Sto00, Theorem 2.10]).

In [Lec06, Lec07] the author gives a deterministic algorithm for the multivariate rational factorization. The rational factorization of a polynomial $f$ is the factorization in $\mathbb{K}[\underline{X}]$, where $\mathbb{K}$ is the coefficient field of $f$. This algorithm uses one factorization of a univariate polynomial of degree $d$ and $\tilde{\mathcal{O}}\left(d^{n+\omega-1}\right)$ arithmetic operations, where $d$ is the total degree of the polynomial and $n \geq 2$ is the number of variables.

Main theorem. The following theorem gives the complexity result of our algorithm.

Theorem 1. Let $f$ be a multivariate rational function in $\mathbb{Q}[\alpha]\left(X_{1}, \ldots, X_{n}\right)$ of degree $d$, where $\alpha$ is an algebraic number of degree $r$. Under hypotheses $(C)$ and $(H)$, we can compute in a deterministic way the decomposition of $f$ with $\tilde{\mathcal{O}}\left(r d^{n+\omega-1}\right)$ arithmetic operations over $\mathbb{Q}$ plus two factorizations of univariate polynomials of degree $d$ with coefficients in $\mathbb{Q}[\alpha]$.

Comparison with other algorithms. There already exist several algorithms for the decomposition of rational functions. They all use the same global strategy: first compute $h$, and then deduce $u$. The first step is the difficult part of the problem. 
In Chè10, we explain how we can perform the second step, i.e., compute $u$ from $h$ and $f$, with $\tilde{\mathcal{O}}\left(d^{n}\right)$ arithmetic operations.

In GRS01, the authors provide two algorithms to decompose a multivariate rational function. These algorithms run in exponential time in the worst case. In the first algorithm we have to factorize polynomials with $2 n$ variables $f_{1}(\underline{X}) f_{2}(\underline{Y})$ $-f_{1}(\underline{Y}) f_{2}(\underline{X})$ and to look for factors of the following kind $h_{1}(\underline{X}) h_{2}(\underline{Y})$ $-h_{1}(\underline{Y}) h_{2}(\underline{X})$. The authors say that in the worst case the number of candidates to be tested is exponential in $d=\operatorname{deg}\left(f_{1} / f_{2}\right)$. Indeed, the authors test all the possible factors.

In the second algorithm, for each pair of factors $\left(h_{1}, h_{2}\right)$ of $f_{1}$ and $f_{2}$ (i.e., $h_{1}$ divides $f_{1}$ and $h_{2}$ divides $f_{2}$ ), we have to test if there exists $u \in \mathbb{K}(T)$ such that $f_{1} / f_{2}=u\left(h_{1} / h_{2}\right)$. Thus in the worst case we also have an exponential number of candidates to be tested.

To the author's knowledge, the first polynomial time algorithm is due to J. Moulin Ollagnier; see MO04. This algorithm relies on the study of the kernel of the following derivation: $\delta_{\omega}(F)=\omega \wedge d F$, where $F \in \mathbb{K}[\underline{X}]$ and $\omega=f_{2} d f_{1}-f_{1} d f_{2}$. In MO04] the author shows that we can reduce the decomposition of a rational function to linear algebra. The bottleneck of this algorithm is the computation of the kernel of a matrix. The size of this matrix is $\mathcal{O}\left(d^{n}\right) \times \mathcal{O}\left(d^{n}\right)$, then the complexity of this deterministic algorithm belongs to $\mathcal{O}\left(d^{n \omega}\right)$.

The reduction of the decomposition problem to a factorization problem is classical; see e.g. Klü99, Gie88, GW95, GRS01]. In [Chè10 the author shows that if we choose a probabilistic approach, then two factorizations in $\mathbb{K}\left[X_{1}, \ldots, X_{n}\right]$ are sufficient to get $h$ and, furthermore, we do not have a recombination problem. This gives a nearly optimal algorithm. For the deterministic approach the author uses a property on the pencil $f_{1}-\lambda f_{2}$ and shows that with $\mathcal{O}\left(d^{2}\right)$ absolute factorization (i.e., factorization in the algebraic closure of $\mathbb{K}$ ) we can get $h$. This deterministic strategy works with $\tilde{\mathcal{O}}\left(d^{n+\omega+2}\right)$ arithmetic operations.

In this paper, we are going to show that we can obtain a deterministic algorithm with just two factorizations in $\mathbb{K}\left[X_{1}, \ldots, X_{n}\right]$ and a recombination strategy. Our algorithm uses at most $\tilde{\mathcal{O}}\left(d^{n+\omega-1}\right)$ arithmetic operations. This cost corresponds to the cost of the factorization and the recombination step.

Our recombination problem comes from the following factorization: If $f_{1} / f_{2}=$ $u_{1} / u_{2}\left(h_{1} / h_{2}\right)$, then

$$
f_{1}-\lambda f_{2}=e\left(h_{1}-t_{1} h_{2}\right) \cdots\left(h_{1}-t_{k} h_{2}\right)
$$

where $\lambda, e \in \mathbb{K}, k=\operatorname{deg}\left(u_{1} / u_{2}\right)$ and $t_{i}$ are the roots of the univariate polynomial $u_{1}(T)-\lambda u_{2}(T)$; see Lemma 10.

Thus with the factors $h_{1}-t_{1} h_{2}$ and $h_{1}-t_{2} h_{2}$ we can deduce $h$. Unfortunately these factors are not necessarily in $\mathbb{K}\left[X_{1}, \ldots, X_{n}\right]$ and are not necessarily irreducible. In this paper we show how we can reduce the problem to a factorization problem in $\mathbb{K}\left[X_{1}, \ldots, X_{n}\right]$ and how we can recombine the irreducible factors of $f_{1}-\lambda f_{2}$ to get $h$.

We can see our recombination scheme as a logarithmic derivative method. Roughly speaking, the logarithmic derivative method works as follows:

If $F(X, Y)=\prod_{j=1}^{t} \mathcal{F}_{j}(X, Y)$, where $\mathcal{F}_{j}(X, Y) \in \mathbb{A}$ and $\mathbb{A} \supset \mathbb{K}[X, Y]$ (for example, $\mathbb{A}=\mathbb{K}[[X]][Y])$, then we can write the irreducible factors $F_{i}(X, Y) \in \mathbb{K}[X, Y]$ of $F$ in the following way: $F_{i}=\prod_{j=1}^{t} \mathcal{F}_{j}^{e_{i, j}}$, where $e_{i, j} \in\{0,1\}$. Thus we just have 
to compute the exponents $e_{i, j}$ to deduce $F_{i}$. We compute these exponents thanks to this relation:

$$
\frac{\partial_{X} F_{i}}{F_{i}}=\sum_{j=1}^{t} e_{i, j} \frac{\partial_{X} \mathcal{F}_{j}}{\mathcal{F}_{j}} .
$$

With this relation the exponents $e_{i, j}$ are now coefficients, and we can compute them with linear algebra.

This strategy has already been used by several authors in order to factorize polynomials; see e.g. BHKS09, BLS ${ }^{+} 04$, Lec06, CL07, Wei10]. Here, we use this kind of technique for the decomposition problem. With this strategy the recombination part of our algorithm corresponds to the computation of the kernel of a $\mathcal{O}\left(d^{n}\right) \times \mathcal{O}(d)$ matrix.

In our context, we do not use exactly a logarithmic derivative. We use a more general derivation, but we use the same idea: If a mathematical object transforms a product into a sum, then the recombination problem becomes a linear algebra problem. In this paper this mathematical object is the cofactor; see Proposition 8

Structure of this paper. In Section 1, we recall some results about the Jacobian derivative and Darboux polynomials. In Section 2, we describe a reduction step which eases the recombination strategy. In other words, we explain how we can reduce the decomposition problem to a factorization problem. In Section 3, we explain how we can get $h$ with a recombination strategy. In Section 4 , we describe our algorithm with two examples. In Section 5 we conclude this paper with a remark on Darboux method and the logarithmic derivative method. In a first appendix, we explain how the strategy proposed recently by J. Berthomieu and G. Lecerf in [BL10] for the sparse factorization can be used in the decomposition setting. Then we deduce a decomposition algorithm in the sparse bivariate case and we give its complexity. In a second appendix, we study the bit-complexity of our algorithm.

Notations. All rational functions are supposed to be reduced.

Given a polynomial $f, \operatorname{deg}(f)$ denotes its total degree.

Given a rational function $f=f_{1} / f_{2}, \operatorname{deg}(f)$ denotes $\max \left(\operatorname{deg}\left(f_{1}\right), \operatorname{deg}\left(f_{2}\right)\right)$.

For the sake of simplicity, sometimes we write $\mathbb{K}[\underline{X}]$ instead of $\mathbb{K}\left[X_{1}, \ldots, X_{n}\right]$, for $n \geq 2$.

$u \circ h$ means $u(h)$.

$\operatorname{Res}(A, B)$ denotes the resultant of two univariate polynomials $A$ and $B$.

$|S|$ is the cardinal of the set $S$.

\section{Derivation and Darboux polynomials}

We introduce the main tool of our algorithm.

Definition 2. A $\mathbb{K}$-derivation $D$ of the polynomial ring $\mathbb{K}\left[X_{1}, \ldots, X_{n}\right]$ is a $\mathbb{K}$-linear map from $\mathbb{K}\left[X_{1}, \ldots, X_{n}\right]$ to itself that satisfies the Leibniz rule for the product

$$
D(f . g)=D(f) \cdot g+f \cdot D(g) .
$$

A $\mathbb{K}$-derivation has a unique extension to $\mathbb{K}\left(X_{1}, \ldots, X_{n}\right)$ and then we will also denote by $D$ the extended derivation. 
Definition 3. Given a rational function $f_{1} / f_{2}$, the Jacobian derivative associated to $f_{1} / f_{2}$ is the following vector derivation, i.e., an $(n-1)$-tuple of derivations:

$$
\begin{aligned}
D_{f_{1} / f_{2}}: \mathbb{K}\left[X_{1}, \ldots, X_{n}\right] & \longrightarrow\left(\mathbb{K}\left[X_{1}, \ldots, X_{n}\right]\right)^{n-1} \\
F & \longmapsto f_{2}^{2} \cdot\left(\begin{array}{c}
\partial_{X_{1}}\left(f_{1} / f_{2}\right) \partial_{X_{2}} F-\partial_{X_{2}}\left(f_{1} / f_{2}\right) \partial_{X_{1}} F \\
\vdots \\
\partial_{X_{1}}\left(f_{1} / f_{2}\right) \partial_{X_{n}} F-\partial_{X_{n}}\left(f_{1} / f_{2}\right) \partial_{X_{1}} F
\end{array}\right) .
\end{aligned}
$$

The Jacobian derivative has the following property:

Proposition 4. Given $f=f_{1} / f_{2}$ and $g \in \mathbb{K}\left(X_{1}, \ldots, X_{n}\right) \backslash \mathbb{K}$ the following propositions are equivalent:

(1) The rank of the Jacobian matrix

$$
J a c(f, g)=\left(\begin{array}{ccc}
\frac{\partial f}{\partial X_{1}} & \cdots & \frac{\partial f}{\partial X_{n}} \\
\frac{\partial g}{\partial X_{1}} & \cdots & \frac{\partial g}{\partial X_{n}}
\end{array}\right)
$$

is equal to one;

(2) $D_{f_{1} / f_{2}}(g)=0$;

(3) there exists $h$ in $\mathbb{K}\left(X_{1}, \ldots, X_{n}\right)$ such that $f=u(h)$ and $g=v(h)$ for $u, v \in \mathbb{K}(T)$.

Proof. See PI07] for a proof. In PI07, $\mathbb{K}$ is supposed to be algebraically closed. However, we can remove this hypothesis because we have the equivalence: $f$ is composite over $\mathbb{K}$ if and only if $f$ is composite over $\overline{\mathbb{K}}$; see e.g. $\overline{B C N}$, Theorem $13]$.

Definition 5. Given $D$ a vector derivation i.e. an $m$-tuple of derivations, a polynomial $F \in \mathbb{K}[\underline{X}]$ is said to be a Darboux polynomial of $D$ if there exists $\mathcal{G} \in(\mathbb{K}[\underline{X}])^{m}$ such that $D(F)=F \cdot \mathcal{G}$. $\mathcal{G}$ is called the cofactor of $F$ for the derivation $D$.

We deduce easily the following classical propositions.

Proposition 6. $f_{1}$ and $f_{2}$ are Darboux polynomials of $D_{f_{1} / f_{2}}$.

Proposition 7. $D_{f_{1} / f_{2}}\left(h_{1} / h_{2}\right)=0$ if and only if $h_{1}$ and $h_{2}$ are Darboux polynomials with the same cofactor.

The following proposition is the main tool of our algorithm. Indeed, this proposition shows that cofactors transform a product into a sum. Then thanks to the cofactors it will be possible to apply a kind of logarithmic derivative recombination scheme.

Proposition 8. Let $F \in \mathbb{K}\left[X_{1}, \ldots, X_{n}\right]$ be a polynomial and let $F=F_{1}^{e_{1}} \cdots F_{r}^{e_{r}}$ be its irreducible factorization in $\mathbb{K}\left[X_{1}, \ldots, X_{n}\right]$. Then:

$F$ is a Darboux polynomial with cofactor $\mathcal{G}_{F}$ if and only if all the $F_{i}$ are Darboux polynomials with cofactor $\mathcal{G}_{F_{i}}$. Furthermore, $\mathcal{G}_{F}=e_{1} \mathcal{G}_{F_{1}}+\cdots+e_{r} \mathcal{G}_{F_{r}}$.

Proof. See, for example, Lemma 8.3 page 216 in DLA06. 


\section{REDUCTION TO A RATiOnAL FACTORIZATION PROBLEM}

In this section, we recall how the decomposition problem can be reduced to a factorization problem. Furthermore, we show that we can reduce our problem to a situation where $f_{1}$ and $f_{2}$ are squarefree. First, we recall some useful lemmas.

Lemma 9. If $f_{1} / f_{2}$ is reduced in $\mathbb{K}\left(X_{1}, \ldots, X_{n}\right)$, where $n \geq 1$ and $\Lambda$ is a variable, then $f_{1}+\Lambda f_{2}$ is squarefree.

Lemma 10. Let $h=h_{1} / h_{2}$ be a rational function in $\mathbb{K}(\underline{X}), u=u_{1} / u_{2}$ a rational function in $\mathbb{K}(T)$ and set $f=u \circ h$ with $f=f_{1} / f_{2} \in \mathbb{K}(\underline{X})$. For all $\lambda \in \mathbb{K}$ such that $\operatorname{deg}\left(u_{1}-\lambda u_{2}\right)=\operatorname{deg} u$, we have

$$
f_{1}-\lambda f_{2}=e\left(h_{1}-t_{1} h_{2}\right) \cdots\left(h_{1}-t_{k} h_{2}\right)
$$

where $e \in \mathbb{K}, k=\operatorname{deg} u$ and $t_{i}$ are the roots of the univariate polynomial $u_{1}(T)$ $\lambda u_{2}(T)$.

Proof. See Chè10, Lemma 8, Lemma 39].

Lemma 11. If $\lambda=f_{1}(\underline{a}) / f_{2}(\underline{a})$, where $\underline{a}=\left(a_{1}, \ldots, a_{n}\right) \in \mathbb{K}^{n}$, then we can suppose that $t_{1} \in \mathbb{K}$.

Proof. We just have to remark that $t_{1}=h_{1}(\underline{a}) / h_{2}(\underline{a}) \in \mathbb{K}$ is a root of $u_{1}-\lambda u_{2}$.

The following lemma says that we can always suppose that $\operatorname{deg} u_{1}=\operatorname{deg} u_{2}=$ $\operatorname{deg} u$.

Lemma 12. Let $h=h_{1} / h_{2}$ be a rational function in $\mathbb{K}(\underline{X}), u=u_{1} / u_{2}$ a rational function in $\mathbb{K}(T)$ and set $f=u \circ h$ with $f=f_{1} / f_{2} \in \mathbb{K}(\underline{X})$. There exists an homography $H(T)=(a T+b) /(\alpha T+\beta) \in \mathbb{K}(T)$ such that:

$u \circ H=\tilde{u}_{1} / \tilde{u}_{2}, \operatorname{deg} \tilde{u}_{1}=\operatorname{deg} \tilde{u}_{2}$, and $f=\frac{\tilde{u}_{1}}{\tilde{u}_{2}} \circ \tilde{h}$, where $\tilde{h}=H^{-1} \circ h$ and $H^{-1}$ is the inverse of $H$ for the composition.

Proof. If $\operatorname{deg} u_{1}=\operatorname{deg} u_{2}$, then we set $H(T)=T$.

If $\operatorname{deg} u_{2}>\operatorname{deg} u_{1}$, then we have

$$
\frac{u_{1}}{u_{2}}(H(T))=\frac{\prod_{i=1}^{\operatorname{deg} u_{1}}\left(a T+b-\lambda_{i}(\alpha T+\beta)\right)}{\prod_{i=1}^{\operatorname{deg} u_{2}}\left(a T+b-\mu_{i}(\alpha T+\beta)\right)} \cdot(\alpha T+\beta)^{\operatorname{deg} u_{2}-\operatorname{deg} u_{1}},
$$

where $u_{1}\left(\lambda_{i}\right)=0$ and $u_{2}\left(\mu_{i}\right)=0$. We set

$$
\begin{aligned}
\tilde{u}_{1}(T) & =(\alpha T+\beta)^{\operatorname{deg} u_{2}-\operatorname{deg} u_{1}} \cdot \prod_{i=1}^{\operatorname{deg} u_{1}}\left(a T+b-\lambda_{i}(\alpha T+\beta)\right) \\
& =u_{1}(H(T)) \cdot(\alpha T+\beta)^{\operatorname{deg} u_{2}} \in \mathbb{K}[T], \\
\tilde{u}_{2}(T) & =\prod_{i=1}^{\operatorname{deg} u_{2}}\left(a T+b-\mu_{i}(\alpha T+\beta)\right) \\
& =u_{2}(H(T)) \cdot(\alpha T+\beta)^{\operatorname{deg} u_{2}} \in \mathbb{K}[T] .
\end{aligned}
$$

If $a-\lambda_{i} \alpha \neq 0, \alpha \neq 0$, and $a-\mu_{i} \alpha \neq 0$, then we get $\operatorname{deg} \tilde{u}_{1}=\operatorname{deg} u_{2}=\operatorname{deg} \tilde{u}_{2}$.

To conclude the proof we just have to remark that $\operatorname{deg} H=1$, thus $H$ is invertible for the composition. 
In order to ease the recombination scheme we reduce our problem to a situation where the rational function is squarefree, i.e., the numerator and the denominator are squarefree. The following algorithm shows that if $f_{1}$ or $f_{2}$ are not squarefree, then we can compute an homography $U(T) \in \mathbb{K}(T)$ such that $U\left(f_{1} / f_{2}\right)$ is squarefree. Furthermore, if we know a decomposition $U\left(f_{1} / f_{2}\right)=u(h)$, then we can easily deduce a decomposition $f_{1} / f_{2}=U^{-1}(u(h))$. We recall that $U$ is invertible for the composition because $\operatorname{deg} U=1$. Now, we describe an algorithm which computes a good homography.

\section{Good Homography}

Input: $f=f_{1} / f_{2} \in \mathbb{K}\left(X_{1}, \ldots, X_{n}\right)$ of degree $d$, such that $(\mathrm{C})$ and $(\mathrm{H})$ are satisfied and a finite subset $S$ of $\mathbb{K}^{n}$ such that $|S|=2 d^{2}+2 d$.

Output: $U(T)=\left(T-\lambda_{a}\right) /\left(T-\lambda_{b}\right)$ such that $U(f)$ is squarefree, $\lambda_{a}=$ $f_{1} / f_{2}(\underline{a}), \lambda_{b}=f_{1} / f_{2}(\underline{b})$ where $\underline{a}, \underline{b} \in \mathbb{K}^{n}, \lambda_{a} \neq \lambda_{b}$, and $\operatorname{deg}_{X_{n}}\left(f_{1}-\lambda_{a} f_{2}\right)=$ $\operatorname{deg}_{X_{n}}\left(f_{1}-\lambda_{b} f_{2}\right)=d$.

(1) Compute $\bar{f}_{1}\left(X_{n}\right):=f_{1}\left(\underline{0}, X_{n}\right)$, and $\bar{f}_{2}\left(X_{n}\right):=f_{2}\left(\underline{0}, X_{n}\right)$.

(2) Construct an empty list $L$.

(3) For $i$ from 1 to $2 d^{2}+2 d$ do:

(a) Compute $\bar{f}:=\bar{f}_{1}(i) / \bar{f}_{2}(i)$.

(b) If $\bar{f} \notin L$, then $L:=$ concatenate $(L,[\bar{f}])$.

(4) Construct an empty list $\mathcal{L}$.

(5) For $k$ from 1 to $2 d+2$ do:

(a) Compute

$$
R:=\operatorname{Res}_{X_{n}}\left(\bar{f}_{1}\left(X_{n}\right)-L[k] \bar{f}_{2}\left(X_{n}\right), \partial_{X_{n}} \bar{f}_{1}\left(X_{n}\right)-L[k] \partial_{X_{n}} \bar{f}_{2}\left(X_{n}\right)\right) .
$$

(b) If $R \neq 0$ and $\operatorname{deg}_{X_{n}}\left(\bar{f}_{1}-L[k] \bar{f}_{2}\right)=d$, then $\mathcal{L}:=\operatorname{concatenate}(\mathcal{L},[L[k]])$.

(6) $\lambda_{a}:=\mathcal{L}[1], \lambda_{b}:=\mathcal{L}[2]$.

(7) Return $U(T)=\left(T-\lambda_{a}\right) /\left(T-\lambda_{b}\right)$.

Proposition 13. The algorithm Good Homography is correct.

Proof. In Step 3 we construct a list with at least $2 d+2$ distinct elements because $\operatorname{deg}(f)=d$.

By hypothesis $(\mathrm{H}), R(\Lambda) \neq 0$ and by [GG03, Theorem 6.22], $\operatorname{deg}(R) \leq 2 d-1$. Thus $\mathcal{L}$ contains at least two distinct elements.

As $R\left(\lambda_{a}\right)$ and $R\left(\lambda_{b}\right)$ are not equal to zero, and thanks to Step (5b) the condition on the degree is satisfied, we deduce that $f_{1}-\lambda_{a} f_{2}$ and $f_{1}-\lambda_{b} f_{2}$ are squarefree.

Proposition 14. The algorithm Good Homography can be performed with at most $\tilde{\mathcal{O}}\left(d^{n}\right)$ arithmetic operations over $\mathbb{K}$.

Proof. Step 1 can be done with $\tilde{\mathcal{O}}\left(d^{n}\right)$ arithmetic operations with Horner's method. In Step 3 we use a fast multipoint evaluation strategy, then we can perform this step with at most $\tilde{\mathcal{O}}\left(d^{2}\right)$ arithmetic operations; see [GG03, Corollary 10.8].

In Step 5 , the computation of the resultant can be done with $\tilde{\mathcal{O}}(d)$ arithmetic operations; see GG03, Corollary 11.16]. Thus Step 5 can be done with $\tilde{\mathcal{O}}\left(d^{2}\right)$ arithmetic operations.

In conclusion the algorithm can be performed with the desired complexity.

Lemma 15. Suppose $f_{1} / f_{2}=v_{1} / v_{2}(h)$. With the algorithm Good Homography we can write $U\left(f_{1} / f_{2}\right)=u_{1} / u_{2}(h)$ with $u_{1} / u_{2} \in \mathbb{K}(T), h \in \mathbb{K}\left(X_{1}, \ldots, X_{n}\right)$, and $u_{1}$ (resp. $u_{2}$ ) has a root $\alpha_{1}$ (resp. $\left.\alpha_{2}\right)$ in $\mathbb{K}$. 
Proof. We have $u_{1}=v_{1}-\lambda_{a} v_{2}$ (resp. $u_{2}=v_{1}-\lambda_{b} v_{2}$ ) and $\lambda_{a}=f_{1} / f_{2}(\underline{a})$ (resp. $\left.\lambda_{b}=f_{1} / f_{2}(\underline{b})\right)$, then we deduce that $\alpha_{1}=h_{1} / h_{2}(\underline{a})\left(\operatorname{resp} . \alpha_{2}=h_{1} / h_{2}(\underline{b})\right)$.

\section{THE RECOMBINATION METHOD}

In this section we describe our recombination method. First, we introduce some notation. By Proposition 6, $F_{1}$ and $F_{2}$ are Darboux polynomials of $D_{F_{1} / F_{2}}$. We denote by

$$
\mathcal{G}_{F_{k}}=\left(\mathcal{G}_{F_{k}}^{(2)}, \ldots, \mathcal{G}_{F_{k}}^{(n)}\right)
$$

the cofactor of $F_{k}$, where $k=1,2$, and $\mathcal{G}_{F_{k}}^{(l)} \in \mathbb{K}\left[X_{1}, \ldots, X_{n}\right]$. We set

$$
F_{k}=\prod_{j=1}^{s_{k}} F_{k, j}
$$

for $k=1,2$, and

$$
\mathcal{G}_{F_{k, j}}=\left(\mathcal{G}_{F_{k, j}}^{(2)}, \ldots, \mathcal{G}_{F_{k, j}}^{(n)}\right) \text {. }
$$

In $\mathbb{Q}[\alpha]\left[X_{1}, \ldots, X_{n}\right]$ polynomials are denoted in the following way:

$$
\mathcal{P}=\sum_{|\tau| \leq d} \sum_{\epsilon=0}^{r-1} a_{\epsilon, \tau} \alpha^{\epsilon} X_{1}^{\tau_{1}} \cdots X_{n}^{\tau_{n}} \in \mathbb{Q}[\alpha]\left[X_{1}, \ldots, X_{n}\right],
$$

where $\alpha$ is an algebraic number of degree $r, \tau=\left(\tau_{1}, \ldots, \tau_{n}\right),|\tau|=\tau_{1}+\cdots+\tau_{n}$, and $a_{\epsilon, \tau} \in \mathbb{Q}$. We set

$$
\operatorname{coef}\left(\mathcal{P}, \alpha^{\epsilon} \underline{X}^{\tau}\right)=a_{\epsilon, \tau} .
$$

Now we define the linear system $\mathcal{S}$ :

$$
\mathcal{S}:=\sum_{j=1}^{s_{1}} x_{1, j} \operatorname{coef}\left(\mathcal{G}_{F_{1, j}}^{(l)}, \alpha^{\epsilon} \underline{X}^{\tau}\right)-\sum_{j=1}^{s_{2}} x_{2, j} \operatorname{coef}\left(\mathcal{G}_{F_{2, j}}^{(l)}, \alpha^{\epsilon} \underline{X}^{\tau}\right)=0,
$$

where $|\tau| \leq d, 0 \leq \epsilon \leq r-1$, and $2 \leq l \leq n$.

We denote by $\operatorname{ker} \mathcal{S}$ the kernel of this linear system, and we remark that

$$
x=\left(x_{1,1}, \ldots, x_{2, s_{2}}\right) \in \operatorname{ker} \mathcal{S} \Longleftrightarrow \sum_{j=1}^{s_{1}} x_{1, j} \mathcal{G}_{F_{1, j}}-\sum_{j=1}^{s_{2}} x_{2, j} \mathcal{G}_{F_{2, j}}=0 .
$$

We define the following maps:

$$
\begin{aligned}
\pi_{1}: \mathbb{K}^{s_{1}+s_{2}} & \longrightarrow \mathbb{K}^{s_{1}} \\
\left(x_{1,1}, \ldots, x_{2, s_{2}}\right) & \longmapsto\left(x_{1,1}, \ldots, x_{1, s_{1}}\right) \\
\pi_{2}: \mathbb{K}^{s_{1}+s_{2}} & \longrightarrow \mathbb{K}^{s_{2}} \\
\left(x_{1,1}, \ldots, x_{2, s_{2}}\right) & \longmapsto\left(x_{2,1}, \ldots, x_{2, s_{2}}\right)
\end{aligned}
$$

The following proposition will be the key of our algorithm:

Proposition 16. Suppose that $F_{1} / F_{2} \in \mathbb{K}\left(X_{1}, \ldots, X_{n}\right)$ comes from the algorithm Good Homography and $F_{1} / F_{2}=u(h)$ where $h=h_{1} / h_{2} \in \mathbb{K}\left(X_{1}, \ldots, X_{n}\right)$ is a noncomposite reduced rational function and $u=u_{1} / u_{2} \in \mathbb{K}(T)$ is a reduced rational function, with $\operatorname{deg} u_{1}=\operatorname{deg} u_{2}$.

We denote by $u_{k}=\prod_{i=1}^{t_{k}} u_{k, i}$ the factorization of $u_{k}$ in $\mathbb{K}[T]$, where $k=1,2$.

We denote by $F_{k}=\prod_{j=1}^{\bar{s}_{k}} F_{k, j}$ the factorization of $F_{k}$ in $\mathbb{K}\left[X_{1}, \ldots, X_{n}\right]$, where $k=1,2$. 
Then:

(1) $u_{k, i}\left(\frac{h_{1}}{h_{2}}\right) \cdot h_{2}^{\operatorname{deg} u_{k, i}}=\prod_{j=1}^{s_{k}} F_{k, j}^{e_{k, i, j}} \in \mathbb{K}\left[X_{1}, \ldots, X_{n}\right]$ and $e_{k, i, j} \in\{0,1\}$.

Furthermore, if we set $e_{k, i}:=\left(e_{k, i, 1}, \ldots, e_{k, i, s_{k}}\right)$, then

the vectors $e_{k, i}, i=1, \ldots, t_{k}$, are orthogonal for the usual scalar product.

(2) We have $e_{k, i} \in \pi_{k}(\operatorname{ker} \mathcal{S})$.

(3) $\left\{e_{k, 1}, \ldots, e_{k, t_{k}}\right\}$ is a basis of $\pi_{k}(\operatorname{ker} \mathcal{S})$.

Proof. (1) By Lemma 10 applied to $F_{1} / F_{2}$ (resp. $F_{2} / F_{1}$ ) with $\lambda=0$, we get

$$
F_{k}=u_{k}\left(h_{1} / h_{2}\right) \cdot h_{2}^{\operatorname{deg} u_{k}}=\prod_{i=1}^{t_{k}} u_{k, i}\left(h_{1} / h_{2}\right) \cdot h_{2}^{\operatorname{deg} u_{k, i}} .
$$

Then we deduce

$$
u_{k, i}\left(h_{1} / h_{2}\right) \cdot h_{2}^{\operatorname{deg} u_{k, i}}=\prod_{j=1}^{s_{k}} F_{k, j}^{e_{k, i}} \text { in } \mathbb{K}\left[X_{1}, \ldots, X_{n}\right]
$$

with $e_{k, i, j} \in\{0,1\}$ because $F_{k}$ are squarefree. Furthermore, the vectors $e_{k, i}$ are orthogonal for the usual scalar product because $F_{k}$ are squarefree.

(2) We show this item for $k=1$, the case $k=2$ can be proved in a similar way.

As $F_{1} / F_{2}$ comes from the algorithm Good Homography and as explained in Lemma 15 we can suppose that:

$$
u_{k, 1}(T)=\left(T-\alpha_{k}\right), \text { with } \alpha_{k} \in \mathbb{K} .
$$

The previous item allows us to write:

$$
\frac{u_{1, i}}{u_{2,1}^{\operatorname{deg} u_{1, i}}}\left(\frac{h_{1}}{h_{2}}\right)=\frac{\left(\prod_{j=1}^{s_{1}} F_{1, j}^{e_{1, i, j}}\right) \cdot\left(h_{2}\right)^{\operatorname{deg} u_{1, i}}}{\left(\prod_{j=1}^{s_{2}} F_{2, j}^{e_{2,1, j}}\right)^{\operatorname{deg} u_{1, i}} \cdot\left(h_{2}\right)^{\operatorname{deg} u_{1, i}}}=\frac{\prod_{j=1}^{s_{1}} F_{1, j}^{e_{1, i, j}}}{\left(\prod_{j=1}^{s_{2}} F_{2, j}^{e_{2,1, j}}\right)^{\operatorname{deg} u_{1, i}}} .
$$

By Proposition 4 applied to $\frac{u_{1, i}}{u_{2,1}^{\operatorname{deg} u_{1, i}}}\left(\frac{h_{1}}{h_{2}}\right)$, we then get:

$$
D_{F_{1} / F_{2}}\left(\frac{\prod_{j=1}^{s_{1}} F_{1, j}^{e_{1, i, j}}}{\prod_{j=1}^{s_{2}} F_{2, j}^{e_{2,1, j} \cdot \operatorname{deg} u_{1, i}}}\right)=0 .
$$

Now, we recall that $F_{k, j}$ are Darboux polynomials; see Proposition [6 and Proposition 8. Then by Proposition 8, we deduce

$$
\sum_{j=1}^{s_{1}} e_{1, i, j} \mathcal{G}_{F_{1, j}}-\operatorname{deg}\left(u_{1, i}\right) \sum_{j=1}^{s_{2}} e_{2,1, j} \mathcal{G}_{F_{2, j}}=0
$$

It follows $\left(e_{1, i, 1}, \ldots, e_{1, i, s_{1}}, \operatorname{deg}\left(u_{1, i}\right) \cdot e_{2,1,1}, \ldots, \operatorname{deg}\left(u_{1, i}\right) \cdot e_{2,1, s_{2}}\right) \in \operatorname{ker} \mathcal{S}$. Thus, $e_{1, i} \in \pi_{1}(\operatorname{ker} \mathcal{S})$.

(3) The vectors $e_{k, 1}, \ldots, e_{k, t_{k}}$ are linearly independent because they are orthogonal. We just have to prove that these vectors generate $\pi_{k}(\operatorname{ker} \mathcal{S})$.

Suppose that $\rho=\left(\rho_{1}, \ldots, \rho_{s_{1}+s_{2}}\right) \in \operatorname{ker} \mathcal{S}$. First, we clear the denominators and we suppose that $\rho \in \mathbb{Z}^{s_{1}+s_{2}}$ instead of $\mathbb{Q}^{s_{1}+s_{2}}$. In a first time we explain the strategy of the proof for this item, and in a second time we will detail the proof. 
We set

$$
\frac{\mathcal{F}_{1}}{\mathcal{F}_{2}}=\frac{\prod_{i=1}^{s_{1}} F_{1, j}^{\rho_{j}}}{\prod_{j=1}^{s_{2}} F_{2, j}^{\rho_{s_{1}+j}}},
$$

where $\mathcal{F}_{1}, \mathcal{F}_{2} \in \mathbb{K}[\underline{X}]$ and $\mathcal{F}_{1} / \mathcal{F}_{2}$ is a reduced rational function.

Our goal is to get this kind of equality:

$$
(\mathcal{E}), \frac{\mathcal{F}_{1}}{\mathcal{F}_{2}}=\frac{\prod_{j=1}^{s_{1}} F_{1, j}^{\rho_{j}}}{\prod_{j=1}^{s_{2}} F_{2, j}^{\rho_{s_{1}+j}}}=\frac{\prod_{k=1}^{2} \prod_{(i, k) \in I_{\mathrm{num}}}\left(\prod_{j=1}^{s_{k}} F_{k, j}^{e_{k, i, j}}\right)^{m_{u_{k, i}}}}{\prod_{k=1}^{2} \prod_{(i, k) \in I_{\mathrm{den}}}\left(\prod_{j=1}^{s_{k}} F_{k, j}^{e_{k, i, j}}\right)^{m_{u_{k, i}}}},
$$

where $m_{u_{k, i}} \in \mathbb{N}, I=\left\{(1,1), \ldots,\left(t_{1}, 1\right),(1,2), \ldots,\left(t_{2}, 2\right)\right\}, I_{\text {num }} \subset I, I_{\text {den }} \subset I$ and $I_{\text {num }} \cap I_{\text {den }}=\emptyset$.

By the unicity of the factorization in irreducible factors we deduce:

$$
\begin{aligned}
& \pi_{1}(\rho)=\sum_{(i, 1) \in I_{\text {num }}} m_{u_{1, i}} e_{1, i}-\sum_{(i, 1) \in I_{\mathrm{den}}} m_{u_{1, i}} e_{1, i}, \\
& \pi_{2}(\rho)=\sum_{(i, 2) \in I_{\text {num }}} m_{u_{2, i}} e_{2, i}-\sum_{(i, 2) \in I_{\mathrm{den}}} m_{u_{2, i}} e_{2, i} .
\end{aligned}
$$

We get that $\left\{e_{k, 1}, \ldots, e_{k, t_{k}}\right\}$ generates $\pi_{k}(\operatorname{ker} \mathcal{S})$, and this is the desired result. Now we detail the proof with four steps:

(a) We remark:

$$
\frac{F_{1}}{F_{2}}=\frac{u_{1}}{u_{2}}(h)=\frac{\prod_{i=1}^{\operatorname{deg} u}\left(h_{1}-\mu_{1, i} h_{2}\right)}{\prod_{i=1}^{\operatorname{deg} u}\left(h_{1}-\mu_{2, i} h_{2}\right)},
$$

where $\mu_{k, i}$ are roots of $u_{k}$.

(b) We have:

$$
\frac{\mathcal{F}_{1}}{\mathcal{F}_{2}}=\frac{\prod_{j=1}^{d_{1}}\left(h_{1}-\lambda_{j} h_{2}\right)^{m_{j}}}{\prod_{j=d_{1}+1}^{d_{1}+d_{2}}\left(h_{1}-\lambda_{j} h_{2}\right)^{m_{j}}} \cdot h_{2}^{\kappa}, \text { with } \kappa \in \mathbb{Z}, m_{j} \in \mathbb{N} .
$$

Indeed, as $\rho \in \operatorname{ker} \mathcal{S}$, we have

$$
\sum_{j=1}^{s_{1}} \rho_{j} \mathcal{G}_{F_{1, j}}-\sum_{j=1}^{s_{2}} \rho_{s_{1}+j} \mathcal{G}_{F_{2, j}}=0 .
$$

Thus $\prod_{j=1}^{s_{1}} F_{1, j}^{\rho_{j}}$ and $\prod_{j=1}^{s_{2}} F_{2, j}^{\rho_{s_{1}+j}}$ are Darboux polynomials with the same cofactor. By Proposition [7, we deduce:

$$
D_{F_{1} / F_{2}}\left(\frac{\prod_{j=1}^{s_{1}} F_{1, j}^{\rho_{j}}}{\prod_{j=1}^{s_{2}} F_{2, j}^{\rho_{s_{1}+j}}}\right)=0 .
$$

Then $D_{F_{1} / F_{2}}\left(\mathcal{F}_{1} / \mathcal{F}_{2}\right)=0$ and thus $\mathcal{F}_{1} / \mathcal{F}_{2}=v_{1} / v_{2}(h)$ by Proposition 4 . We denote by $\lambda_{j}$ the roots of $v_{1}$ and $v_{2}$ and we get the desired result.

(c) We claim:

$$
\frac{\mathcal{F}_{1}}{\mathcal{F}_{2}}=\frac{\prod_{k=1}^{2} \prod_{(i, k) \in I_{\text {num }}}\left(u_{k, i}\left(h_{1} / h_{2}\right) h_{2}^{\operatorname{deg} u_{k, i}}\right)^{m_{u_{k, i}}}}{\prod_{k=1}^{2} \prod_{(i, k) \in I_{\mathrm{den}}}\left(u_{k, i}\left(h_{1} / h_{2}\right) h_{2}^{\operatorname{deg} u_{k, i}}\right)^{m_{u_{k, i}}}}, \text { where } m_{u_{k}, i} \in \mathbb{N} \text {. }
$$


Indeed, we have for all $j$ there exists $\delta(j)$ such that $\lambda_{j}=\mu_{\delta(j)}$.

(To prove this remark we suppose the converse: There exists $j_{0}$ such that $\lambda_{j_{0}} \neq$ $\mu_{k, i}$, for $k=1,2$ and $i=1, \ldots, \operatorname{deg} u$.

By definition of $\mathcal{F}_{k}$ and by step (3) $)$, there exists $\left(k_{1}, j_{1}\right)$ such that $F_{k_{1}, j_{1}}$ and $h_{1}-\lambda_{j_{0}} h_{2}$ have a common factor in $\mathbb{C}[\underline{X}]$. We call $\mathcal{P}$ this common factor.

By step (3a), there exists $\left(k_{2}, i_{2}\right)$ such that $\mathcal{P}$ is a factor of $h_{1}-\mu_{k_{2}, i_{2}} h_{2}$.

Thus $h_{1}-\lambda_{j_{0}} h_{2}$ and $h_{1}-\mu_{k_{2}, i_{2}} h_{2}$ have a common factor. As $\lambda_{j_{0}} \neq \mu_{k_{2}, i_{2}}$ we deduce that $\mathcal{P}$ divides $h_{1}$ and $h_{2}$. This is absurd because $h_{1} / h_{2}$ is reduced.)

Thus $\kappa=0$, and for all $j$ there exists $k(j) \in\{1,2\}$ and such that $u_{k(j)}\left(\lambda_{j}\right)=0$.

As $v_{1}, v_{2} \in \mathbb{K}[T]$, by conjugation, we deduce that if $\lambda_{j}$ and $\lambda_{j^{\prime}}$ are roots of the same irreducible polynomial $u_{k, i} \in \mathbb{K}[T]$, then $m_{j}=m_{j^{\prime}}$.

We denote by $m_{u_{k, i}}$ this common value.

This gives the claimed equality with $I_{\text {num }} \cap I_{\text {den }}=\emptyset$, because $\mathcal{F}_{1} / \mathcal{F}_{2}$ is reduced.

(d) Now we can prove equality $(\mathcal{E})$.

$$
\begin{aligned}
\frac{\mathcal{F}_{1}}{\mathcal{F}_{2}}= & \left.\frac{\prod_{k=1}^{2} \prod_{(i, k) \in I_{\mathrm{num}}}\left(u_{k, i}\left(h_{1} / h_{2}\right) h_{2}^{\operatorname{deg} u_{k, i}}\right)^{m_{u_{k}, i}}}{\prod_{k=1}^{2} \prod_{(i, k) \in I_{\mathrm{den}}}\left(u_{k, i}\left(h_{1} / h_{2}\right) h_{2}^{\operatorname{deg} u_{k, i}}\right)^{m_{u_{k, i}}}}, \text { by step (3r) }\right) \text {, } \\
= & \frac{\prod_{k=1}^{2} \prod_{(i, k) \in I_{\mathrm{num}}}\left(\prod_{j=1}^{s_{k}} F_{k, j}^{e_{k, i, j}}\right)^{m_{u_{k, i}}}}{\prod_{k=1}^{2} \prod_{(i, k) \in I_{\mathrm{den}}}\left(\prod_{j=1}^{s_{k}} F_{k, j}^{e_{k, i, j}}\right)^{m_{u_{k, i}}}}, \text { by the first item. }
\end{aligned}
$$

This gives the desired equality $(\mathcal{E})$.

Now we describe our recombination algorithm:

\section{Recombination for Decomposition}

Input: $f=f_{1} / f_{2} \in \mathbb{K}\left(X_{1}, \ldots, X_{n}\right)$, such that $(\mathrm{C})$ and $(\mathrm{H})$ are satisfied.

Output: A decomposition of $f$ if it exists, with $f=u \circ h, u=u_{1} / u_{2}$ with $\operatorname{deg} u \geq 2$, and $h=h_{1} / h_{2}$ non-composite.

(1) Compute $F=F_{1} / F_{2}:=U(f)$ with the algorithm Good Homography.

(2) For $\mathrm{k}=1,2$, factorize $F_{k}=\prod_{i=1}^{s_{k}} F_{k, i}$ in $\mathbb{K}[\underline{X}]$ with $F_{k, i}$ irreducible.

(3) For each $F_{k, i}$ compute the corresponding cofactor $\mathcal{G}_{F_{k, i}}:=D_{F_{1} / F_{2}}\left(F_{k, i}\right) / F_{k, i}$.

(4) Build the system $\mathcal{S}$ and compute the basis in reduced row echelon form $\mathcal{B}_{1}$ of $\pi_{1}(\operatorname{ker} \mathcal{S})$ and $\mathcal{B}_{2}$ of $\pi_{2}(\operatorname{ker} \mathcal{S})$.

(5) For $\mathrm{k}=1$, 2, find $v_{k}=\left(v_{k, 1}, \ldots, v_{k, s_{k}}\right) \in \mathcal{B}_{k}$ such that:

$\sum_{i=1}^{s_{k}} v_{k, i} \operatorname{deg} F_{k, i}=\min _{w \in \mathcal{B}_{k}} \sum_{i=1}^{s_{k}} w_{i} \operatorname{deg} F_{k, i}$, where $w:=\left(w_{1}, \ldots, w_{s_{k}}\right)$.

(6) For $\mathrm{k}=1,2$, compute $H_{k}:=\prod_{i=1}^{s_{k}} F_{k, i}^{v_{k, i}}$.

(7) Set $H:=H_{1} / H_{2}$.

(8) Compute $u$ such that $u(H)=f$.

(9) Return $H$, and $u$.

Proposition 17. The algorithm Recombination for Decomposition is correct.

Proof. Consider $F_{1} / F_{2}:=U(f)$. As we want to decompose $f_{1} / f_{2}$, we just have to decompose $F_{1} / F_{2}$, because $\operatorname{deg} U=1$ and then $U$ is invertible.

As $F_{1} / F_{2}$ comes from the algorithm Good Homography we can suppose (see Lemma [15), that $u_{k, 1}(T)=\left(T-\alpha_{k}\right)$ with $\alpha_{k} \in \mathbb{K}$, and $k=1,2$. Furthermore, by Lemma 12 we can also suppose that $\operatorname{deg} u_{1}=\operatorname{deg} u_{2}$.

Then by Proposition 16, the basis $\mathcal{B}_{k}$ of $\pi_{k}(\operatorname{ker} \mathcal{S})$ are $\left\{e_{k, 1}, \ldots, e_{k, t_{k}}\right\}$. 
The vector $e_{k, i}$ gives the polynomial $\mathcal{H}_{k, i}=\prod_{j=1}^{s_{k}} F_{k, j}^{e_{k, i, j}}=u_{k, i}(h) h_{2}^{\operatorname{deg} u_{k, i}}$. Furthermore, $\operatorname{deg} \mathcal{H}_{k, i}=\sum_{j=1}^{s_{k}} e_{k, i, j} \operatorname{deg} F_{k, j}=\operatorname{deg} u_{k, i} \operatorname{deg} h$. Thus, in Step 5 ,

$$
\min _{w \in \mathcal{B}_{k}} \sum_{i=1}^{s_{k}} w_{i} \operatorname{deg} F_{k, i}=\operatorname{deg} h,
$$

because this minimum is reached with $e_{k, 1} \in \mathcal{B}_{k}$. Hence $v_{k}$ in Step 6 gives $H_{k}=$ $u_{k, i(k)}(h) h_{2}^{\operatorname{deg} u_{k, i(k)}}$ with $\operatorname{deg} u_{k, i(k)}=1$.

It follows $H=\left(h_{1}-\alpha h_{2}\right) /\left(h_{1}-\beta h_{2}\right)$ with $\alpha, \beta \in \mathbb{K}$. Thus $H=v(h)$ with $\operatorname{deg} v=1$, then the algorithm is correct.

Proposition 18. The algorithm Recombination for Decomposition can be performed with $\tilde{\mathcal{O}}\left(r d^{n+\omega-1}\right)$ arithmetic operations over $\mathbb{Q}$ and two factorizations of univariate polynomials of degree $d$ with coefficients in $\mathbb{K}$.

We recall that in our complexity analysis the number of variables is fixed and the degree $d$ tends to infinity.

Proof. Step 1 uses $\tilde{\mathcal{O}}\left(d^{n}\right)$ arithmetic operations over $\mathbb{K}$ by Proposition 14, thus it uses $\tilde{\mathcal{O}}\left(r d^{n}\right)$ arithmetic operations over $\mathbb{Q}$.

Step 2 uses $\tilde{\mathcal{O}}\left(d^{n+\omega-1}\right)$ arithmetic operations over $\mathbb{K}$ because we can use Lecerf's algorithm; see [Lec07]. Thus we use $\tilde{\mathcal{O}}\left(r d^{n+\omega-1}\right)$ arithmetic operations over $\mathbb{Q}$ and two factorizations of univariate polynomials of degree $d$ with coefficients in $\mathbb{K}$.

In Step 3, we compute $D_{F_{1} / F_{2}}\left(F_{k, i}\right)$, thus we perform $2(n-1)$ multiplications of multivariate polynomials. We can do this with a fast multiplication technique, and then this computation costs $\tilde{\mathcal{O}}\left(n r d^{n}\right)$ arithmetic operations over $\mathbb{Q}$. Then we divide $D_{F_{1} / F_{2}}\left(F_{k, i}\right)$ by $F_{k, i}$. We have to perform $n-1$ exact divisions, thus with a Kronecker substitution we reduce this problem to $n-1$ univariate divisions, and the cost of one such division belongs then to $\tilde{\mathcal{O}}\left(r d^{n}\right)$. As $s_{1}$ and $s_{2}$ are smaller than $d$, Step 3 costs $\tilde{\mathcal{O}}\left(n r d^{n+1}\right)$ arithmetic operations over $\mathbb{Q}$.

Step 4 needs $\tilde{\mathcal{O}}\left(n r d^{n} d^{\omega-1}\right)$ arithmetic operations over $\mathbb{Q}$ with Storjohann's method; see [Sto00, Theorem 2.10]. Indeed, $\mathcal{S}$ has $\mathcal{O}\left((n-1) r d^{n}\right)$ equations and $s_{1}+s_{2}$ unknowns, thus at most $2 d$ unknowns.

Step 5 has a negligible cost because $\operatorname{dim}_{\mathbb{Q}} \pi_{k}(\operatorname{ker} \mathcal{S})=t_{k}$ is smaller than $d$ and $s_{k}$ is also smaller than $d$.

In Step [6] we use a fast multiplication technique and we compute $H_{k}$ with $\tilde{\mathcal{O}}\left(r d^{n}\right)$ arithmetic operations over $\mathbb{Q}$.

Step 8 can be done with $\tilde{\mathcal{O}}\left(r d^{n}\right)$ arithmetic operations over $\mathbb{Q}$; see [Chè10].

Thus the global cost of the algorithm belongs to $\tilde{\mathcal{O}}\left(r d^{n+\omega-1}\right)$ arithmetic operations over $\mathbb{Q}$.

\section{EXAMPLES}

In this section we show the behavior of the algorithm Recombination for Decomposition with two examples. We consider bivariate rational functions with rational coefficients. Thus hypothesis (C) is satisfied.

4.1. $f$ is non-composite. We set:

$f_{1}=\left(1+X+Y^{2}\right)(X+Y)=X+X^{2}+X Y^{2}+Y+Y X+Y^{3}$,

$f_{2}=f_{1}-\left(Y^{2}-X-1\right)(Y-2 X+1)=-X^{2}+3 X Y^{2}+2 Y+2 Y X-Y^{2}+1$. 
We have $\operatorname{deg}\left(f_{1}+\Lambda f_{2}\right)=\operatorname{deg}_{Y}\left(f_{1}+\Lambda f_{2}\right)=3$, and

$$
\begin{aligned}
& \operatorname{Res}_{Y}\left(f_{1}(0, Y)+\Lambda f_{2}(0, Y), \partial_{Y} f_{1}(0, Y)+\Lambda \partial_{Y} f_{2}(0, Y)\right) \\
& \quad=-4-24 \Lambda-92 \Lambda^{2}-64 \Lambda^{3}+8 \Lambda^{4} .
\end{aligned}
$$

Thus hypothesis $(\mathrm{H})$ is satisfied.

The algorithm Good Homography gives: $\lambda_{a}=f_{1}(0,0) / f_{2}(0,0)=0$ and $\lambda_{b}=$ $f_{1}(0,1) / f_{2}(0,1)=1$.

Then

$$
\begin{aligned}
F_{1} & =\left(1+X+Y^{2}\right)(X+Y), \\
F_{1,1} & =1+X+Y^{2}, \\
F_{1,2} & =X+Y, \\
F_{2} & =\left(Y^{2}-X-1\right)(Y-2 X+1), \\
F_{2,1} & =Y^{2}-X-1, \\
F_{2,2} & =Y-2 X+1 .
\end{aligned}
$$

The cofactors are:

$$
\begin{aligned}
& \mathcal{G}_{F_{1,1}}=3 X^{2}+8 Y X^{2}+2 X-2 Y X+7 X Y^{2}-1+3 Y^{2}-6 Y^{3}-6 Y^{4}+2 Y, \\
& \mathcal{G}_{F_{1,2}}=3 X^{2}+8 Y X^{2}+4 Y X+6 X-6 Y^{2}-4 Y+3-3 Y^{4}-2 Y^{3}, \\
& \mathcal{G}_{F_{2,1}}=3 X^{2}+8 Y X^{2}+X Y^{2}-6 Y X+2 X-1-2 Y-6 Y^{4}-11 Y^{2}-6 Y^{3}, \\
& \mathcal{G}_{F_{2,2}}=3 X^{2}+8 Y X^{2}+6 X Y^{2}+8 Y X+6 X-3 Y^{4}+8 Y^{2}-2 Y^{3}+3 .
\end{aligned}
$$

The linear system $\mathcal{S}$ is the following:

$$
\left[\begin{array}{cccc}
-1 & 3 & -1 & 3 \\
2 & 6 & 2 & 6 \\
3 & 3 & 3 & 3 \\
0 & 0 & 0 & 0 \\
0 & 0 & 0 & 0 \\
2 & -4 & -2 & 0 \\
-2 & 4 & -6 & 8 \\
8 & 8 & 8 & 8 \\
0 & 0 & 0 & 0 \\
3 & -6 & -11 & 8 \\
7 & 0 & 1 & 6 \\
0 & 0 & 0 & 0 \\
-6 & -2 & -6 & -2 \\
0 & 0 & 0 & 0 \\
-6 & -3 & -6 & -3
\end{array}\right]
$$

A basis of $\operatorname{ker}(\mathcal{S})$ is given by $\{(-1,-1,1,1)\}$. Then it follows that $f_{1} / f_{2}$ is non-composite. 
4.2. $f$ is composite. Here we set:

$$
\begin{aligned}
h_{1} & =\left(1+X+Y^{2}\right)(X+Y), \\
h_{2} & =h_{1}-\left(Y^{2}-X-1\right)(Y-2 X+1), \\
u_{1} & =T \cdot(T-1), \\
u_{2} & =T^{2}+1 \\
f_{1} / f_{2} & =u_{1} / u_{2}\left(h_{1} / h_{2}\right) .
\end{aligned}
$$

We have constructed a composite rational function $f_{1} / f_{2}$ and now we illustrate how our algorithm computes a decomposition. We can already remark that in the previous example we have shown that $h_{1} / h_{2}$ is non-composite.

In this situation the hypothesis $(\mathrm{H})$ is satisfied and the algorithm Good Homography gives: $\lambda_{a}=f_{1}(0,0) / f_{2}(0,0)=0$ and $\lambda_{b}=f_{1}(0,2) / f_{2}(0,2)=90 / 101$.

Then:

$$
\begin{aligned}
& F_{1,1}=1+X+Y^{2} \\
& F_{1,2}=2 X-Y-1 \\
& F_{1,3}=Y^{2}-X-1 \\
& F_{1,4}=X+Y \\
& F_{2,1}=2 X^{2}+11 X+9+29 X Y+29 Y+38 X Y^{2}-9 Y^{2}+11 Y^{3} \\
& F_{2,2}=11 X^{2}+X-10-19 Y X-19 Y-29 X Y^{2}+10 Y^{2}+Y^{3}
\end{aligned}
$$

The basis in reduced row echelon form of $\pi_{1}(\operatorname{ker} \mathcal{S})$ (respectively $\pi_{2}($ ker $\mathcal{S})$ ) is $\{(1,0,0,1) ;(0,1,1,0)\}$ (respectively $\{(1,0) ;(0,1)\}$ ).

Step [5] in the algorithm Recombination for Decomposition give: $v_{1}=(1,0,0,1)$ and $v_{2}=(1,0)$.

Then we have $H_{1}:=F_{1,1} \cdot F_{1,4}$ and $H_{2}:=F_{2,1}$.

We remark that $H_{1}=h_{1}$ and that $H_{2}=11 h_{1}+9 h_{2}$. Then $H_{1} / H_{2}=w\left(h_{1} / h_{2}\right)$, where $w(T)=T /(11 T+9)$. As $h_{1} / h_{2}$ is non-composite and $\operatorname{deg} w=1$, we get a correct output.

\section{Conclusion}

In conclusion, we summarize our algorithm with a "derivation point of view".

In order to decompose $f_{1} / f_{2}$, we have computed with Darboux method a rational first integral of $D_{f_{1} / f_{2}}$ with minimum degree. That is to say we have computed $h_{1} / h_{2} \in \mathbb{K}\left(X_{1}, \ldots, X_{n}\right)$ such that $D_{f_{1} / f_{2}}\left(h_{1} / h_{2}\right)=0$ and $\operatorname{deg}\left(h_{1} / h_{2}\right)$ is minimum. In a general setting, Darboux method works as follows: If we want to compute a rational first integral of a derivation $D$, first we compute all the Darboux polynomials $F_{i}$ and their associated cofactors $\mathcal{G}_{F_{i}}$, second we solve the linear system

$$
\sum_{i} e_{i} \mathcal{G}_{F_{i}}=0
$$

Then thanks to Proposition 8, we deduce that $\prod_{i} F_{i}^{e_{i}}$ is a first integral, i.e.,

$$
D\left(\prod_{i} F_{i}^{e_{i}}\right)=0 .
$$

When we consider the derivation $D_{f_{1} / f_{2}}$ the computation of Darboux polynomials is reduced to the factorization of $f_{1}+\lambda f_{2}$. Thus this step can be done efficiently. 
In the general setting, we can also reduce the computation of Darboux polynomials to a factorization problem; see Chè11.

During the second step, we compute the kernel of $\sum_{i} e_{i} \mathcal{G}_{F_{i}}=0$. It is actually a recombination step. Indeed, this system explains how we have to recombine $F_{i}$ in order to get a rational first integral. Furthermore, the cofactor $\mathcal{G}_{F_{i}}=D\left(F_{i}\right) / F_{i}$ can be viewed as a logarithmic derivative.

In conclusion, the recombination scheme used in this paper is called nowadays the logarithmic derivative method, but this method is Darboux original method.

\section{Appendix A. ConveX-Dense Bivariate Decomposition}

In this appendix we give complexity results for the decomposition of sparse bivariate rational functions. These results rely on a strategy proposed by J. Berthomieu and G. Lecerf in BL10].

Given a polynomial $f(X, Y) \in \mathbb{K}[X, Y]$, its support is the set $S_{f}$ of integer points $(i ; j)$ such that the monomial $X^{i} Y^{j}$ appears in $f$ with a nonzero coefficient. The convex hull, in the real space $\mathbb{R}^{2}$ of $S_{f}$ is denoted by $N(f)$ and called the Newton's polygon of $f$. We denote by $|N(f)|$ the number of integral points of $N(f)$. We called $|N(f)|$ the convex-size of $f$.

Roughly speaking, the transformation proposed in BL10 consists in a monomial transformation that preserves the convex-size but decreases the dense size. The considered transformation $\mathcal{T}$ can be described in the following way:

$$
\begin{gathered}
\mathcal{T}=\mathcal{B} \circ \mathcal{L}, \text { where } \\
\mathcal{B}\left(X^{i} Y^{j}\right)=X^{i+b_{1}} Y^{j+b_{2}}, b_{1}, b_{2} \in \mathbb{Z}, \\
\mathcal{L}\left(X^{i} Y^{j}\right)=X^{a_{1} i+a_{2} j} Y^{a_{3} i+a_{4} j}, a_{1} a_{4}-a_{2} a_{3}= \pm 1 .
\end{gathered}
$$

$\mathcal{T}$ can be defined on $\mathbb{K}\left[X, Y, X^{-1}, Y^{-1}\right]$, and we define: $\mathcal{T}\left(\sum_{i, j} f_{i, j} X^{i} Y^{j}\right)=$ $\sum_{i, j} f_{i, j} \mathcal{T}\left(X^{i} Y^{j}\right)$

The transformation $\mathcal{L}$ corresponds to the linear map: $(i, j) \mapsto \mathcal{A}^{t}(i, j)$, where

$$
\mathcal{A}=\left(\begin{array}{ll}
a_{1} & a_{2} \\
a_{3} & a_{4}
\end{array}\right) .
$$

We denote by $\mathcal{L}^{-1}$ the transformation corresponding to $\mathcal{A}^{-1}$.

If $f(X, Y) \in \mathbb{K}[X, Y]$, then $\mathcal{L}(f) \in \mathbb{K}\left[X, Y, X^{-1}, Y^{-1}\right]$ and $\mathcal{L}(f)$ can be written $\mathcal{L}(f)=c_{\mathcal{L}}(f) . \mathcal{L}_{0}(f)$, where $\mathcal{L}_{0}(f) \in \mathbb{K}[X, Y]$ and $c_{\mathcal{L}}(f)=X^{i} Y^{j} \in \mathbb{K}\left[X, Y, X^{-1}, Y^{-1}\right]$. Furthermore, we also have $\mathcal{L}\left(F_{1} \cdot F_{2}\right)=\mathcal{L}\left(F_{1}\right) \cdot \mathcal{L}\left(F_{2}\right)$.

Let $S$ be a finite subset of $\mathbb{Z}^{2}$. Set $S$ is said to be normalized if it belongs to $\mathbb{N}^{2}$ and if it contains at least one point in $\{0\} \times \mathbb{N}$, and also at least one point in $\mathbb{N} \times\{0\}$. For such a normalized set, we write $d_{x}$ (resp. $d_{y}$ ) for the largest abscissa (resp. ordinate) involved in $S$, so that the bounding rectangle is $\mathcal{R}=\left[0, d_{x}\right] \times\left[0, d_{y}\right]$. The following result is proved in [BL10, Theorem 2]:

For any normalized finite subset $S$ of $\mathbb{Z}^{2}$, of cardinality $\sigma$, convex-size $\pi$, and bounding rectangle $\left[0, d_{x}\right] \times\left[0, d_{y}\right]$, and dense size $\delta=\left(d_{x}+1\right)\left(d_{y}+1\right)$, one can compute an affine map $\mathcal{T}=\mathcal{B} \circ \mathcal{L}$, with $\mathcal{O}\left(\sigma \log ^{2} \delta\right)$ bit-operations, such that $\mathcal{T}(S)$ is normalized of dense size at most $9 \pi$.

We are going to use this transformation in order to prove:

Theorem 19. Let $f_{1} / f_{2}(X, Y) \in \mathbb{K}(X, Y)$ such that $\operatorname{deg}\left(f_{1} / f_{2}\right)=d, N\left(f_{1}\right) \subset N$, $N\left(f_{2}\right) \subset N$ and $N$ is normalized. Then: 
(1) If $\mathbb{K}$ is a field with characteristic 0 or at least $d(d-1)+1$ and (H) is satisfied, then there exists a probabilistic algorithm which computes the decomposition of $f_{1} / f_{2}$ with at most $\tilde{\mathcal{O}}\left(|N|^{1,5}\right)$ operations in $\mathbb{K}$ and two factorizations of a univariate polynomial of degree at most $9|N|$ over $\mathbb{K}$.

(2) If $(C)$ and (H) are satisfied, then there exists a deterministic algorithm which computes the decomposition of $f_{1} / f_{2}$ with at most $\tilde{\mathcal{O}}\left(r .|N|^{(\omega+1) / 2}\right)$ operations over $\mathbb{Q}$ and two factorizations of an univariate polynomials of degree at most $9|N|$ over $\mathbb{Q}[\alpha]$.

Now, we explain how we use the transformation $\mathcal{T}$ in the decomposition setting.

Proposition 20. If $f_{1} / f_{2}=u\left(h_{1} / h_{2}\right)$, then $\mathcal{T}\left(f_{1}\right) / \mathcal{T}\left(f_{2}\right)=u\left(\mathcal{L}\left(h_{1}\right) / \mathcal{L}\left(h_{2}\right)\right)$.

If $\mathcal{T}\left(f_{1}\right) / \mathcal{T}\left(f_{2}\right)=u\left(H_{1} / H_{2}\right)$, then $f_{1} / f_{2}=u\left(\mathcal{L}^{-1}\left(H_{1}\right) / \mathcal{L}^{-1}\left(H_{2}\right)\right)$.

Proof. We prove the first item, the second can be proved in a similar way.

We have $\frac{f_{1}}{f_{2}}=\frac{\prod_{i}\left(h_{1}-\mu_{1, i} h_{2}\right)}{\prod_{j}\left(h_{1}-\mu_{2, j} h_{2}\right)}$, where $\mu_{k, i}$ are roots of $u_{k}$. Then,

This gives the following algorithm:

$$
\begin{aligned}
\frac{\mathcal{T}\left(f_{1}\right)}{\mathcal{T}\left(f_{2}\right)} & =\frac{\mathcal{B} \circ \mathcal{L}\left(f_{1}\right)}{\mathcal{B} \circ \mathcal{L}\left(f_{2}\right)}=\frac{X^{b_{1}} Y^{b_{2}} \mathcal{L}\left(f_{1}\right)}{X^{b_{1}} Y^{b_{2}} \mathcal{L}\left(f_{2}\right)} \\
& =\frac{\mathcal{L}\left(f_{1}\right)}{\mathcal{L}\left(f_{2}\right)}=\frac{\prod_{i}\left(\mathcal{L}\left(h_{1}\right)-\mu_{1, i} \mathcal{L}\left(h_{2}\right)\right)}{\prod_{j}\left(\mathcal{L}\left(h_{1}\right)-\mu_{2, j} \mathcal{L}\left(h_{2}\right)\right)} \\
& =\frac{u_{1}\left(\mathcal{L}\left(h_{1}\right) / \mathcal{L}\left(h_{2}\right)\right)}{u_{2}\left(\mathcal{L}\left(h_{1}\right) / \mathcal{L}\left(h_{2}\right)\right)}=u\left(\mathcal{L}\left(h_{1}\right) / \mathcal{L}\left(h_{2}\right)\right) .
\end{aligned}
$$

\section{Convex Bivariate Decomposition}

Input: $f=f_{1} / f_{2} \in \mathbb{K}(X, Y)$, where $N\left(f_{1}\right) \subset N, N\left(f_{2}\right) \subset N$ and $N$ is normalized.

Output: A decomposition of $f$ if it exists, with $f=u \circ h, u=u_{1} / u_{2}$ with $\operatorname{deg} u \geq 2$, and $h=h_{1} / h_{2}$ non-composite.

(1) Compute $F=\mathcal{T}\left(f_{1}\right) / \mathcal{T}\left(f_{2}\right)$.

(2) Decompose $F=u(H)$.

(3) Return $f=u(h)$, where $h=\frac{\mathcal{L}^{-1}\left(H_{1}\right)}{\mathcal{L}^{-1}\left(H_{2}\right)}=\frac{c_{\mathcal{L}^{-1}}\left(H_{1}\right) \cdot \mathcal{L}_{0}^{-1}\left(H_{1}\right)}{c_{\mathcal{L}^{-1}}\left(H_{2}\right) \cdot \mathcal{L}_{0}^{-1}\left(H_{2}\right)} \in \mathbb{K}(X, Y)$.

Proposition 21. The algorithm Convex Bivariate Decomposition is correct.

Proof. This follows from Proposition 20.

Proposition 22. The algorithm Convex Bivariate Decomposition uses one decomposition of a rational function of degree at most $9|N|$ and $\mathcal{O}\left(\sigma^{2} \delta\right)$ bit operations.

Proof. We apply BL10, Theorem 2] to $N$.

The proof of Theorem 19 comes from Proposition 21 and Proposition 22 and complexity results given in Theorem 1 and Chè10, Theorem 2].

\section{Appendix B. Bit-COMplexity}

In this paper we have shown that we can improve Moulin Ollagnier's algorithm. We have measured this improvement with the arithmetic complexity, but a natural question arises: what can be said about the bit-complexity? 
In this appendix, we describe briefly a modular strategy for the algorithm Recombination for Decomposition. In order to study the complexity of this algorithm we suppose that we have a fast arithmetic, that is to say we can compute $a \times b$, $a / b, a+b$ and $a-b$ with $\tilde{\mathcal{O}}(l)$ bit-operations where $l$ is the bit-size of $a$ and $b$; see GG03, Theorem 8.24].

We consider $f$ a multivariate rational function in $\mathbb{Z}\left(X_{1}, \ldots, X_{n}\right)$ of degree $d$ and height $\mathcal{H}$. That is to say, $f_{1}$ and $f_{2}$ belong to $\mathbb{Z}\left[X_{1}, \ldots, X_{n}\right]$ and their heights are bounded by $\mathcal{H}$.

First we remark that we can suppose $h_{1}$ and $h_{2}$ in $\mathbb{Z}\left[X_{1}, \ldots, X_{n}\right]$.

Indeed, if $h_{i}=H_{i} / d_{i}$ where $H_{i} \in \mathbb{Z}\left[X_{1}, \ldots, X_{n}\right]$ and $d_{i} \in \mathbb{Z}$, then we have $f=u\left(d_{2} / d_{1} \cdot H_{1} / H_{2}\right)$. Thus, with $v(T)=u\left(d_{2} / d_{1} . T\right)$ we have $f=v\left(H_{1} / H_{2}\right)$ and $H_{i} \in \mathbb{Z}\left[X_{1}, \ldots, X_{n}\right]$

Now, we modify slightly the algorithm Good Homography. Instead of computing $U\left(f_{1} / f_{2}\right)$ with $U(T)=(T-f(\underline{a})) /(T-f(\underline{b}))$ we compute $V\left(f_{1} / f_{2}\right)$ with $V(T)=$ $\left(f_{2}(\underline{a}) T-f_{1}(\underline{a})\right) /\left(f_{2}(\underline{b}) T-f_{1}(\underline{b})\right)$. We set $F_{1} / F_{2}=V\left(f_{1} / f_{2}\right)$. Now $F_{1}$ (resp. $\left.F_{2}\right)$ belongs to $\mathbb{Z}\left[X_{1}, \ldots, X_{n}\right]$ and has a factor $h_{2}(\underline{a}) h_{1}-h_{1}(\underline{a}) h_{2}\left(\right.$ resp. $h_{2}(\underline{b}) h_{1}-$ $\left.h_{1}(\underline{b}) h_{2}\right)$ in $\mathbb{Z}\left[X_{1}, \ldots, X_{n}\right]$.

As $\underline{a}=(0, \ldots, 0, x)$ where $x \leq 2 d^{2}+2 d$, we get a rough estimate for $f_{i}(\underline{a})$ :

$$
\left|f_{i}(\underline{a})\right| \leq(d+1) H\left(2 d^{2}+2 d\right)^{d} .
$$

Then, we get

$$
\mathcal{H}\left(F_{k}\right) \leq 2(d+1) H^{2}\left(2 d^{2}+2 d\right)^{d},
$$

where $\mathcal{H}\left(F_{k}\right)$ is the height of $F_{k}$.

A classical result about the height of the factors of a polynomials (see Sch00, Corollary 12 p. 249]) gives

$$
\mathcal{H}\left(F_{k, i}\right) \leq 2^{n d^{2}} \mathcal{H}\left(F_{k}\right),
$$

where $\mathcal{H}\left(F_{k, i}\right)$ is the height of $F_{k, i}$. Then we deduce:

$$
\mathcal{H}\left(F_{k, i}\right) \leq 2^{n d^{2}+1}(d+1) \mathcal{H}^{2}\left(2 d^{2}+2 d\right)^{d}<e^{n d^{2}+1+2 d \ln (2 d)}(d+1) \mathcal{H}^{2}=\mathcal{B}_{1} .
$$

Thus, if we factorize $F_{k}$ in $\mathbb{F}_{p_{1}}\left[X_{1}, \ldots, X_{n}\right]$, where $p_{1}$ is a prime such that $4 \mathcal{B}_{1}+2 \geq p_{1}>2 \mathcal{B}_{1}+1$, then we can compute $F_{k, i}$ with $\tilde{\mathcal{O}}\left(d^{n+\omega-1} \ln \left(\mathcal{B}_{1}\right)\right)$ bit-operations. The factor $\ln \left(\mathcal{B}_{1}\right)$ is the bit-size of $p_{1}$. Then this step needs $\tilde{\mathcal{O}}\left(d^{n+\omega-1} n d^{2} \ln (\mathcal{H})\right)$ bit-operations. We can rewrite this complexity estimate in the following way: $\tilde{\mathcal{O}}\left(n d^{n+\omega+1} \ln (\mathcal{H})\right)$.

A direct computation shows that $\mathcal{H}(A B) \leq(\operatorname{deg} A+\operatorname{deg} B)^{n} \mathcal{H}(A) \mathcal{H}(B)$, where $A, B \in \mathbb{Z}\left[X_{1}, \ldots, X_{n}\right]$. In our context this gives:

$$
\mathcal{H}\left(D_{F_{1} / F_{2}}\left(F_{k, i}\right)\right) \leq 2(3 d-2)^{n}(2 d-1)^{n} d \mathcal{H}^{2} \mathcal{B}_{1} .
$$

As $\mathcal{G}_{F_{k, i}}$ is a factor of $D_{F_{1} / F_{2}}\left(F_{k, i}\right)$ we deduce as before that

$$
\mathcal{H}\left(\mathcal{G}_{F_{k, i}}\right) \leq 2^{n(2 d-1)} \mathcal{H}\left(D_{F_{1} / F_{2}}\left(F_{k, i}\right)\right) .
$$

Then, it follows that

$$
\mathcal{H}\left(\mathcal{G}_{F_{k, i}}\right) \leq 2 e^{n((2 d-1)+\ln (3 d-2)+\ln (2 d-1))} d \mathcal{H}^{2} \mathcal{B}_{1}=\mathcal{B}_{2} .
$$

With Hadamard's bound we deduce that all the minors of $\mathcal{S}$ are bounded by $d^{d / 2} \mathcal{B}_{2}=\mathcal{B}_{3}$. Thus, if we compute in our algorithm the reduced row echelon form modulo $p_{2}$, where $p_{2}$ is a prime number such that $p_{2} \geq \mathcal{B}_{3}$, then we get a correct output. This computation needs $\tilde{\mathcal{O}}\left(d^{n+\omega-1} \ln \left(\mathcal{B}_{3}\right)\right)$ bit-operations. As $\ln \left(\mathcal{B}_{3}\right)$ belongs 
to $\tilde{\mathcal{O}}\left(n d^{2} \ln (\mathcal{H})\right)$, we conclude that this step can be done with $\tilde{\mathcal{O}}\left(n d^{n+\omega+1} \log (\mathcal{H})\right)$ bit-operations.

Thus there exists a modular version of the algorithm Recombination for Decomposition which works with $\tilde{\mathcal{O}}\left(n d^{n+\omega+1} \log (\mathcal{H})\right)$ bit-operations.

In this estimate we do not have take into account the computation of the big prime $p_{1}$ and $p_{2}$. This cost is negligible if we use a probabilistic approach; see GG03, Theorem 18.8].

As the bit-complexity of Moulin Ollagnier's algorithm is bigger than $\tilde{\mathcal{O}}\left(d^{n \omega}\right)$ we can conclude that the modular version of the algorithm Recombination for Decomposition has a better bit-complexity than Moulin Ollagnier's one when $n \geq 3$.

\section{REFERENCES}

[AGR95] C. Alonso, J. Gutierrez, and T. Recio. A rational function decomposition algorithm by near-separated polynomials. J. Symbolic Comput., 19(6):527-544, 1995. MR 1370620 (96j:13025)

[AT85] V.S. Alagar and Mai Thanh. Fast polynomial decomposition algorithms. In EUROCAL '85, Vol. 2 (Linz, 1985), volume 204 of Lecture Notes in Comput. Sci., pages 150-153. Springer, Berlin, 1985. MR826564

[BCN] L. Busé, G. Chèze, and S. Najib. Noether's forms for the study of non-composite rational functions and their spectrum. A. Arithmetica, 147 (3): 217-231, 2011. MR 2773201

[BDN09] A. Bodin, P. Dèbes, and S. Najib. Indecomposable polynomials and their spectrum. A. Arithmetica, 139(1):79-100, 2009. MR2538746 (2010j:12001)

[BHKS09] K. Belabas, M. van Hoeij, J. Klüners, and A. Steel. Factoring polynomials over global fields. J. Theorie des Nombres de Bordeaux, 21:15-39, 2009. MR2537701(2010d:12001)

[BL10] J. Berthomieu and G. Lecerf. Reduction of bivariate polynomials from convexdense to dense, with application to factorizations. Math. Comp. 81:1799-1821. DOI: http://dx.doi.org/10.1090/S0025-5718-2011-02562-7. MR2904603

$\left[\mathrm{BLS}^{+} 04\right]$ A. Bostan, G. Lecerf, B. Salvy, É. Schost, and B. Wiebelt. Complexity Issues in Bivariate Polynomial Factorization. In Proceedings of ISSAC 2004, pages 42-49. ACM, 2004. MR2126923 (2005k:68084)

[BP94] D. Bini and V.Y. Pan. Polynomial and matrix computations. Vol. 1. Progress in Theoretical Computer Science. Birkhäuser Boston, Inc., Boston, MA, 1994. Fundamental algorithms. MR:1289412 (95k:65003)

[BZ85] D.R. Barton and R. Zippel. Polynomial decomposition algorithms. J. Symbolic Comput., 1(2):159-168, 1985. MR818876 (87b:12001)

[Chè10] G. Chèze. Nearly optimal algorithms for the decomposition of multivariate rational functions and the extended Luroth's theorem. J. Complexity, 26(4):344-363, 2010. MR2669662 (2011g:12006)

[Chè11] G. Chèze. Computation of Darboux polynomials and rational first integrals with bounded degree in polynomial time. J. Complexity, 27 (2): 246-262, 2011. MR2776495

[CL07] G. Chèze and G. Lecerf. Lifting and recombination techniques for absolute factorization. J. Complexity, 23(3):380-420, 2007. MR2330992(2008f:68174)

[CN10] G. Chèze and S. Najib. Indecomposability of polynomials via Jacobian matrix. J. Algebra, 324(1):1-11, 2010. MR2646027 (2011h:12003)

[Dic87] M. Dickerson. Polynomial decomposition algorithms for multivariate polynomials. Technical Report TR87-826, Comput. Sci., Cornell Univ., 1987.

[DLA06] F. Dumortier, J. Llibre, and J.C. Artés. Qualitative theory of planar differential systems. Universitext. Springer-Verlag, Berlin, 2006. MR2256001(2007f:34001)

[FGP10] J.-C. Faugère, J. von zur Gathen, and L. Perret. Decomposition of generic multivariate polynomials. In Proceedings of ISSAC 2010, pages 131-137. ACM, 2010. MR2920546

[FP09] J.-C. Faugère and L. Perret. An efficient algorithm for decomposing multivariate polynomials and its applications to cryptography. J. Symbolic Comput., 44(12):1676-1689, 2009. MR2553573 (2010k:94036)

[Gat90a] J. von zur Gathen. Functional decomposition of polynomials: the tame case. J. Symbolic Comput., 9(3):281-299, 1990. MR.1056628 (92a:12015) 
[Gat90b] J. von zur Gathen. Functional decomposition of polynomials: the wild case. J. Symbolic Comput., 10(5):437-452, 1990. MR1087714(92i:12008)

[Gat11] J. von zur Gathen. Counting decomposable multivariate polynomials. Appl. Algebra Eng. Commun. Comput. 22(3): 165-185, 2011. MR2803912

[GG03] J. von zur Gathen and J. Gerhard. Modern computer algebra. Cambridge University Press, Cambridge, second edition, 2003. MR2001757 (2004g:68202)

[GGR03] J. von zur Gathen, J. Gutierrez, and R. Rubio. Multivariate polynomial decomposition. Appl. Algebra Engrg. Comm. Comput., 14(1):11-31, 2003. MR.1989633 (2004i:12013)

[Gie88] M. Giesbrecht. Some results on the functional decomposition of polynomials. Master Thesis, University of Toronto, arXiv:1004.5433, 1988.

[GRS01] J. Gutierrez, R. Rubio, and D. Sevilla. Unirational fields of transcendence degree one and functional decomposition. In ISSAC '01: Proceedings of the 2001 International Symposium on Symbolic and Algebraic Computation, pages 167-174, New York, NY, USA, 2001. ACM Press. MR2049745 (2005g:12005)

[GW95] J. von zur Gathen and J. Weiss. Homogeneous bivariate decompositions. J. Symbolic Comput., 19(5):409-434, 1995. MR1348781 (96f:12009)

[KL89] D. Kozen and S. Landau. Polynomial decomposition algorithms. J. Symbolic Comput., 7(5):445-456, 1989. MR.999513 (91c:13022)

[Klü99] J. Klüners. On polynomial decompositions. J. Symbolic Comput., 27(3):261-269, 1999. MR1673595 (2000h:12004)

[Lec06] G. Lecerf. Sharp precision in Hensel lifting for bivariate polynomial factorization. Math. Comp., 75(254):921-933 (electronic), 2006. MR2197000 (2007g:12008)

[Lec07] G. Lecerf. Improved dense multivariate polynomial factorization algorithms. J. Symbolic Comput., 42(4):477-494, 2007. MR2317560 (2008e:13031)

[MO04] J. Moulin Ollagnier. Algebraic closure of a rational function. Qual. Theory Dyn. Syst., 5(2):285-300, 2004. MR2275442 (2007h:13035)

[PI07] A.P. Petravchuk and O.G. Iena. On closed rational functions in several variables. Algebra Discrete Math., (2):115-124, 2007. MR.2364068(2009a:26012)

[Rit22] J.F. Ritt. Prime and composite polynomials. Trans. Amer. Math. Soc., 23(1):51-66, 1922. MR 1501189

[Sch00] A. Schinzel. Polynomials with special regard to reducibility, volume 77 of Encyclopedia of Mathematics and its Applications. Cambridge University Press, Cambridge, 2000. With an appendix by Umberto Zannier. MR 1770638 (2001h:11135)

[Sto00] A. Storjohann. Algorithms for matrix canonical forms. PhD thesis, ETH Zurich, Zurich, Switzerland, 2000.

[Wat08] S. Watt. Functional decomposition of symbolic polynomials. In International Conference on Computatioanl Sciences and its Applications, pages 353-362. IEEE Computer Society, 2008 .

[Wat09] S. Watt. Algorithms for the functional decomposition of Laurent polynomials. In Conferences on Intelligent Computer Mathematics 2009: 16th Symposium on the Integration of Symbolic Computation and Mechanized Reasoning and 8th International Conference on Mathematical Knowledge Management, (Calculemus 2009), pages 186-200. Springer-Verlag LNAI 5625, 2009.

[Wei10] M. Weimann. A lifting and recombination algorithm for rational factorization of sparse polynomials. J. Complexity, 26(6):608-628, 2010. MR2735422 (2011j:12008)

[Zip91] R. Zippel. Rational function decomposition. In Proceedings of the 1991 international symposium on Symbolic and algebraic computation, pages 1-6. ACM Press, 1991.

Institut de Mathématiques de Toulouse, Université Paul Sabatier Toulouse 3, MiP BÂt 1R3, 31062 TOULOUSE CEdex 9, France

E-mail address: guillaume.cheze@math.univ-toulouse.fr 